\title{
Perspectives on pediatric endoscopic surgery
}

\author{
Amulya K. Saxena ${ }^{1}$ \\ Published online: 25 February 2019 \\ (c) Springer Nature Singapore Pte Ltd 2019
}

Pediatric minimal access surgery is now a well-established discipline that has progressed from the management of adolescents and children to neonates. There have been many factors that have contributed towards achieving the establishment of endoscopic surgery by enthusiastic pediatric surgeons that have been challenged not only by the pathology, but also by the remarkable variations in size of the pediatric patient. Procedures by pediatric endoscopic surgeons now encompass those performed in the premature and low birth weight neonates as well as those performed in morbid obese adolescents. This variation in size for endoscopic procedures has not only presented challenges to the pediatric surgeons, but also the associated sub-specialties that are involved in the perioperative management and care of these patients bearing in mind that the hemodynamic alterations and body response to insufflation differ considerably with age in the pediatric age group.

Refinements in the size of instruments that were originally conceptualized for procedures in the adults gave the impetus towards the rapid development and acceptance of this approach in the pediatric surgical community. Further improvement to meet the demands of the neonatal surgeons through miniaturization led to the application of endoscopic surgical procedures in neonates and furthermore in premature and low birth weight patients. Improvisations and improvement are still ongoing to scale down the size of instruments to reproduce the benefits seen by the instruments designed for adult use in the pediatric population. With continuous analysis towards improvement of ergonomics, there are ongoing modifications being supported by the industry to provide improved bespoke alternatives for neonatal and pediatric endoscopic surgery.

The implementation of robotics in pediatric endoscopic surgery has opened another avenue in this field. Reports

Amulya K. Saxena

amulya.saxena@nhs.net

1 Chelsea and Westminster Hospital NHS Fdn Trust, Imperial College London, London, UK are continuously emerging with regards to the successful application in the pediatric population. The limitations and the cost analysis are being better understood for the implementation of robots for pediatric patients. There is an enormous hype in the field of mechanical engineering towards the development of more cost effective systems as well as miniaturization of robotic instruments more suitable for neonatal applications. With multiple providers engaged in the development of newer robotic systems, the future trend will involve the comparison between these systems and to equate their advantages in the clinical setting. Not only will the miniaturization of the robotic instruments, but also improvements in the software and ease of handling the hardware consoles, be undergoing the end-user assessments.

Training of pediatric surgeons in endoscopic procedures also requires attention. Various skill labs and pediatric endoscopic surgical hands-on courses have evolved worldwide in the past decades to cater towards introduction and improvement of training. Pelvitrainers and virtual reality trainers have provided the opportunity for trainee surgeons to gain introduction to this field. Live-model skill labs have furthermore enabled the trainee surgeons to perform procedures more specific to pediatric surgery under expert supervision and guidance. Although these courses provide a good insight into pediatric endoscopic surgical procedures, sustained practice in skill labs and continuous performing of these procedures under supervision within one's own institution will be the key to progression from basic procedures to advanced index cases. Incorporation of endoscopic surgery should be integrated in the training program in pediatric surgery and national bodies will have to take decisions on the minimum number of cases that need to be assisted and performed during the training period.

Improvement of ergonomics is an important aspect that will play a continuous role in the development of pediatric endoscopic procedures. These issues will involve the operating suite set-up, positioning of equipment, position and posture of the operating surgeon and their team, positioning of the tubes and cables, instrument handle designs and positioning of the ports to achieve the best outcomes. Continuous 
reporting on ergonomics in the field of endoscopic surgery will be important in performing procedures under optimal circumstance, which in turn will offer enhanced efficiency and safety during surgery. Safety is another issue that will need further addressing as neonatal and pediatric endoscopic procedures are performed in small cavities for which the understanding of manipulation angles and electrosurgical devices will be of paramount importance. Instrument and equipment related injuries are still under reported in the literature, but reporting of such events will raise awareness and decrease the risk of unnecessary untoward outcomes.

Artificial intelligence assisted surgery is presently at its infancy, but it is another area that will make a mark in the foreseeable future of endoscopic surgery. Sophisticated analytics and machine learning methodologies are being developed to assimilate diagnostic insights and published best practices from the countless data elements, and incorporating them into robotic-assisted surgery. Artificial intelligence in endoscopic surgery is envisaged to give a digital standardization by integrating and processing information from three-dimensional imaging, beyond visual depth perception, hemodynamics monitoring and ventilator setting based movement of robotic arms and instruments to achieve a revolutionary paradigm shift in surgery. The advantages of using this technology will mainly focus on the reduction of surgical variations among surgeons and address its associated inadequacies and differences in outcomes.

As we progress towards routinely performing advanced index procedures in neonatal and pediatric patients, outcome evaluation will be imperative to determine the level of evidence in pediatric endoscopic surgery. Over the past three decades there has been a continuous progression in reporting case- and observational studies, but it is now essential to perform systematic review and meta-analysis, by combining multiple observational studies, to understand significances of outcomes. Randomized control trials will also be necessary to determine whether a cause-effect relation exists between treatment and outcome. The perusal of high level of evidence reports, however, should not undermine reporting of rare pathologies that have been managed with innovational endoscopic techniques.

Basic science research is an area where efforts are being devoted to better elucidate the effects of insufflation on pediatric patients. There are multiple uninvestigated areas where the hemodynamic response from endoscopic procedures is poorly understood, especially in premature neonates, neurologically challenged patients and those patients with reduced cerebral, cardiopulmonary or renal function. Since the maximum hemodynamic changes occur during the course of the endoscopic procedures, pediatric anesthesiologists will also appreciate the information from basic research so as to optimize monitoring and anesthesia management in patients with altered compensatory mechanisms. Data also from these studies will optimize selection of above mentioned patients in the pre-operative work-up and assist intensive care teams involved in postoperative care. Endoscopic surgical oncology is another area of basic research where investigations are ongoing to better recognize the effects related to insufflation.

Endoscopic surgery will continue to evolve and modify trends in pediatric practice based on a combination of interlinked advances in technology and surgery. Pediatric endoscopic surgeons will remain on the forefront to apply innovations, technical variations and use of modern instruments and equipment to provide the best services to their young patients. The Journal of Pediatric Endoscopic Surgery is foreseen as a platform to share these advancements among pediatric surgeons and allied health professional as they strive to progress in field of endoscopic surgery.

Amulya K. Saxena

Editor-in-Chief 